\title{
Modified Kaplan-Meier Estimator Based on Competing Risks for Heavy Censoring Data
}

\author{
Ali Zare and Mahmood Mahmoodi*
}

\author{
Department of Epidemiology and Biostatistics, Tehran University of Medical Sciences, Iran
}

\begin{abstract}
Most follow-up studies are conducted to determine the survival rates of subjects affected by a specific risk. These subjects are also exposed to other risks. Every subject in a medical follow-up is exposed not only to the risk of dying, but also to the risk of being censored. In case of heavy censoring, the Kaplan-Meier estimates are biased and overestimate the survival distribution. A new methodology based on competing risks is proposed to estimate the survival function by using net and crude probabilities. These estimates reduce the bias and overestimation of the survival distribution noted in Kaplan-Meier estimators. In this study, the method of modified Kaplan-Meier (MKM) is compared with the Kaplan-Meier (KM), Huang's method and also the two other methods namely Weighted Kaplan-Meier (WKM) and Modified Weighted Kaplan-Meier (MWKM). Either of the weighted methods depends heavily on the event times and censoring distributions. Due to this fact, the weighted methods can have misleading results when the censoring patterns are different in the individual samples. The results showed that the MKM estimator considers not only the problem of heavy censoring but also the problem of weighted methods and competing risks in complicated data. In this study "Stanford Heart Transplant Data" was used to investigate the effectiveness of the proposed methods.
\end{abstract}

Keywords: Competing risks, Kaplan-Meier estimator, Heavy Censoring, Net and Crude probabilities.

\section{INTRODUCTION}

Statistical Methods play an increasingly important role in many fields of medical and allied areas of research. Among these methods, the survival analysis reflects much importance in the analysis of life threatening diseases and other fields of biostatistics. Survival analysis is a collection of statistical procedures for data analysis, for which the outcome variable of interest is the time recorded for an event to occur. The major mathematical complication with survival/failure time data analysis is that one usually does not have the luxury of waiting until the very last subject has died. Therefore one normally has to analyze the data while some subjects are still alive. Moreover, some subjects may have moved away and some are lost in the followup due to other reasons. In both cases, the subjects are known to have survived for some times, known as censored times (i.e. they have been seen until the last time), but one doesn't know, how much longer they may have survived ultimately [1]. Several methods have been developed for constructing survival curve estimates. The most common methods are the Life Table, and Kaplan-Meier methods. These methods rely strongly upon the assumption that the death rate among those subjects under observation (not truncated and not censored) is the same as the death rate in the population of interest [2].

The survival time of an individual is said to be censored, when the event of interest could not be

*Address correspondence to this author at the Department of Epidemiology and Biostatistics, Tehran University of Medical Sciences, Iran; Tel: +9821 88950185; Fax: +9821 88989127; E-mail: mahmoodim@tums.ac.ir recorded for that individual. The reasons for the censored survival time could be the termination of the experiment as in a clinical study it may not be feasible to continue or follow-up the experiment until all the subjects under study have failed or experienced the event of interest or subjects may withdrawn willfully or may getting dropped from the study. In this case it may not be possible to have complete information for all the subjects, Censoring is broadly classified into two categories: Informative and noninformative. In this study we consider informative censoring only. Some of the important types of censoring are Type I censoring (or fixed time censoring), Type II censoring (or fixed number censoring), random censoring and Interval censoring. Type I and Type II are singly censored data where as Type III is random censored data. Type I, Type II and random censoring data are right censored. It is to be noted that when there are no censored observations, the set of survival times is said to be complete [3-4]. High levels of censoring can suggest a number of problems in the study. The Quick end (by which most subjects do not have an outcome at the end of the study) and a pattern of censoring that makes a lot of subjects be excluded from the study in a specific time, are among these problems. Hence, large number of censored observations makes the survival estimations contain error and be estimated higher than their real amounts. Unfortunately, there is no suitable test to determine the validity of censoring assumption, and this is just a judgment made by researchers.

In the case of heavy censoring (a study may be terminated with a large number of censorings, which could be due to loss to follow up, withdrawal, and 
alternative outcome than the focused event), the Kaplan-Meier estimate is not reliable and overestimates the survival probabilities [5]. The KaplanMeier survival curve also fails to give reliable estimates at the end points $[3,6]$. To have a reliable estimate, in the case of heavy censoring, an improved method of Kaplan-Meier estimate, namely Weighted Kaplan-Meier method of estimation was applied and proved reliable estimates by introducing the weights based on the noncensored rate [7, 8]. Then, followed by the Weighted Kaplan-Meier method, a modified form of this, namely Modified Weighted Kaplan-Meier method was introduced by assigning a new weight in the case of the last observation is censored [9]. Then, a Weighted Empirical Survival Function (WESF) was used by Huang's method, in which choices of weights were introduced for obtaining the survival function [10]. Either of the weighted methods depends heavily on the event times and censoring distributions. Due to this fact, the weighted methods can have misleading results when the censoring patterns are different in the individual samples. The new methods based on competing risks are also used for obtaining the survival function by using net and crude probabilities. Finally, in this paper, some conclusions are drawn for the Stanford Heart Transplant Data, by comparing the estimated survival probabilities obtained from the above mentioned methods.

\section{METHODS: KAPLAN-MEIER ESTIMATION}

The Kaplan-Meier estimator is the limit of the life table estimator when intervals are taken so small that at most one observation occurs within an interval [11, 12]. This estimator gives a maximum likelihood estimate.

Suppose the data consists of observed times $t_{1}, \ldots, t_{n}$, including censored observations. In other words, for some of the $t_{j}$, it is only known that individual $j$ was still alive at time $t_{j}$. Let $r$ be the number of distinct failure times, and $t_{(1)} \leq t_{(2)} \leq \ldots \leq t_{(r)}$ be the ordered failure times. Define $n_{j}$ the number of individuals still alive just before $t_{(j)}$ including the individual about to die. Also define $d_{j}$ as the number of individuals who died at time $t_{(j)}$. Then Kaplan-Meier estimator can be expressed as

$$
\hat{s}(t)=\prod_{j t_{(j)} \leq t}\left\{\frac{n_{j}-d_{j}}{n_{j}}\right\}
$$

Note that in the notation above the survival rates change only at the event time. If the last observation is censored, the Kaplan-Meier estimator fails to estimate the tails of the survival function. Furthermore, this method overestimates the survival distribution in case of heavy censoring.

\subsection{Modified Forms of Kaplan-Meier Method of Estimation}

Weighted Kaplan-Meier Estimator of Survival Function:

Jan et al. (2005) claim that in life threatening diseases when a portion of the data is censored, the Kaplan Meier estimator becomes unreliable and inefficient $[7,8]$. To deal with the situation they define a weight $w_{j}$ at time $t_{(j)}$ as

$w_{j}=\left\{\frac{n_{j}-c_{j}}{n_{j}}\right\}$, which is known as non-censored rate.

Where $w_{j}=1$ if there is no censoring and $0<w_{j}<1$ in case of censoring at time $t_{(j)}$ and $c_{j}$ is the number of censored patients at $t_{(j)}$ Then, the Weighted Kaplan-Meier estimator is defined as

$$
s^{*}(t)=\prod_{j t_{(j)} \leq t} w_{j}\left\{\frac{n_{j}-d_{j}}{n_{j}}\right\}
$$

In this case $s^{*}(t)$ will reach zero, if the last observed survival time is censored.

Modified Weighted Kaplan-Meier Estimator:

The main limitation in the above Weighted KaplanMeier method is that it gives zero weight to the last censored observation and probability is equal to zero. To overcome this difficulty, Shafiq et al. (2007) proposed a new weight which gives a non-zero weight to the last censored observation [9]. The proposed Modified Weighted Kaplan-Meier estimator is then,

$$
s^{* *}(t)=\prod_{j t_{(j)} \leq t} w_{j} *\left\{\frac{n_{j}-d_{j}}{n_{j}}\right\}
$$

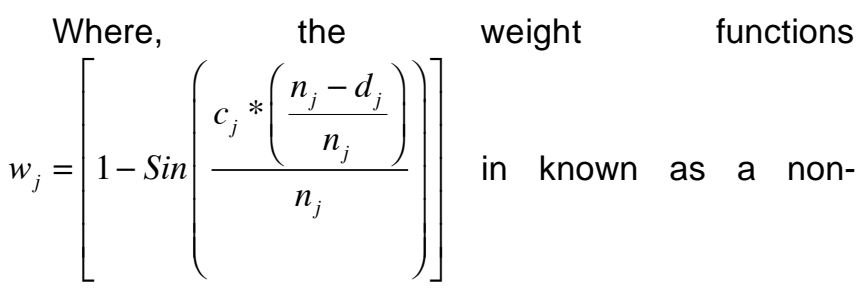


censoring rate. Both the Modified Weighted KaplanMeier and the Weighted Kaplan-Meier estimators give the same weight to all censored observations. They also give the same probability of survival, but the important point is that the Weighted Kaplan-Meier estimator gives zero weight to the last censored observation while the modified weighted estimator gives it some non zero weight and, therefore, has a small probability of survival.

Huang's Estimator of Survival Function:

Huang (2008) studied a Weighted Empirical Survivor Function (WESF) [10]. It has been shown that by choosing appropriate weights, the estimator proposed by him is more efficient than the KaplanMeier estimate in both censored and uncensored data.

For the censoring case, the idea of the above WESF for uncensored case has been applied to obtain a weighted Kaplan-Meier as shown below:

$\hat{s}_{K M W}(t)= \begin{cases}1 \quad, \quad t<\tau_{i} \\ \prod_{i=1}^{j}\left(1-\frac{d_{i}}{n_{i}}\right) *\left(1-p_{k, i}\right) \quad, \quad \tau_{j}<t<\tau_{j+1}, \quad j=1, \ldots ., k-1\end{cases}$

The weights suggested for first, last and other observations by Huang are,

$$
\begin{aligned}
& w \equiv p_{n, i}=\frac{1}{\sqrt{n(n-1)}} ; i=2, \ldots, n-1 \\
& w_{1, n}=p_{n .1}=p_{n, n}=\frac{1}{2} *\left(1-\frac{n-2}{\sqrt{n(n-1)}}\right)
\end{aligned}
$$

where $n$ is the sample size of study.

\section{MODIFIED KAPLAN-MEIER ESTIMATOR BASED ON COMPETING RISKS}

Most follow-up studies are conducted to determine the survival rates of subjects affected by a specific event. These subjects are also exposed to other risks of death from among them, some may eventually die. In a study determining the effectiveness of radiation as a treatment for cancer, for example, some patients may die from heart disease. In such cases, the theory of competing risks is indispensable, and the crude and net probabilities all play roles.

\subsection{Crude and Net Probabilities}

\section{Definitions and Relationships}

Chiang (1961, 1970 and 1991), Bowers (1987) and Jordan (1975) define two types of probabilities [13-17]:
1. Crude probability: the probability of death from a specific cause in the presence of all other risks acting in the population.

$q_{j}^{(\delta)}=\operatorname{Pr}\left\{\right.$ an individual dies in time $t_{j}$ from cause $R_{\delta}$ in the presence of all other risks in the population\}.

2. Net probability: The probability of death if a specific risk is the only risk in effect in the population or, conversely, the probability of death if a specific risk is eliminated from the population.

$q_{j}^{\prime(\delta)}=\operatorname{Pr}$ \{an individual will die in time $t_{j}$ if $R_{\delta}$ is the only risk of death\}.

Assume that $r$ risks denoted by $R_{1}, \ldots, R_{r}$ are acting simultaneously on each patient in the study. An approximate solution and relation for net and crude probability of ding (from risk $R_{1}$ ), is given by the following formula:

$$
q_{j}^{\prime(1)} \frac{q_{j}^{(1)}}{\left[1-0.5\left(q_{j}^{(2)}+\ldots+q_{j}^{(r)}\right)\right]}
$$

More detailed discussion of the methodology is provided by Bowers (1986), Jordan (1975) and Chiang $(1961,1970,1991)$ [13-17]

Every subject in a medical follow-up is exposed not only to the risk of dying, but also to the risk of being censored. Censored subjects have caused difficulties in determining survival rates. For the purpose of determining the probability of dying from a specific cause, censored subjects are not different from those dying of causes unrelated to the study. Being censored, therefore, should be considered as a competing risk, and the survival experience of censored cases should be evaluated by using the methods discussed in this article.

Suppose we let $R_{r}$ denote the risk of being censored. The crude and net probabilities of dying are denoted by:

$q_{j}^{(1)}=\operatorname{Pr}\left\{\right.$ a subject will die at time $t_{j}$ in the presence of $R_{r}$ risk of being censored\}

$q_{j}^{\prime(1)}=\operatorname{Pr}$ \{a subject will die at time $t_{j}$ if the risk $R_{r}$ of being censored is eliminated\} 
Table 1: Survival Probabilities of the HEART TRANSPLANT DATA, Obtained from the K-M Estimate, W-K-M, M-W-K-M, Huang's Method and Modified Kaplan-Meier Estimator Based on Competing Risks (MKM):

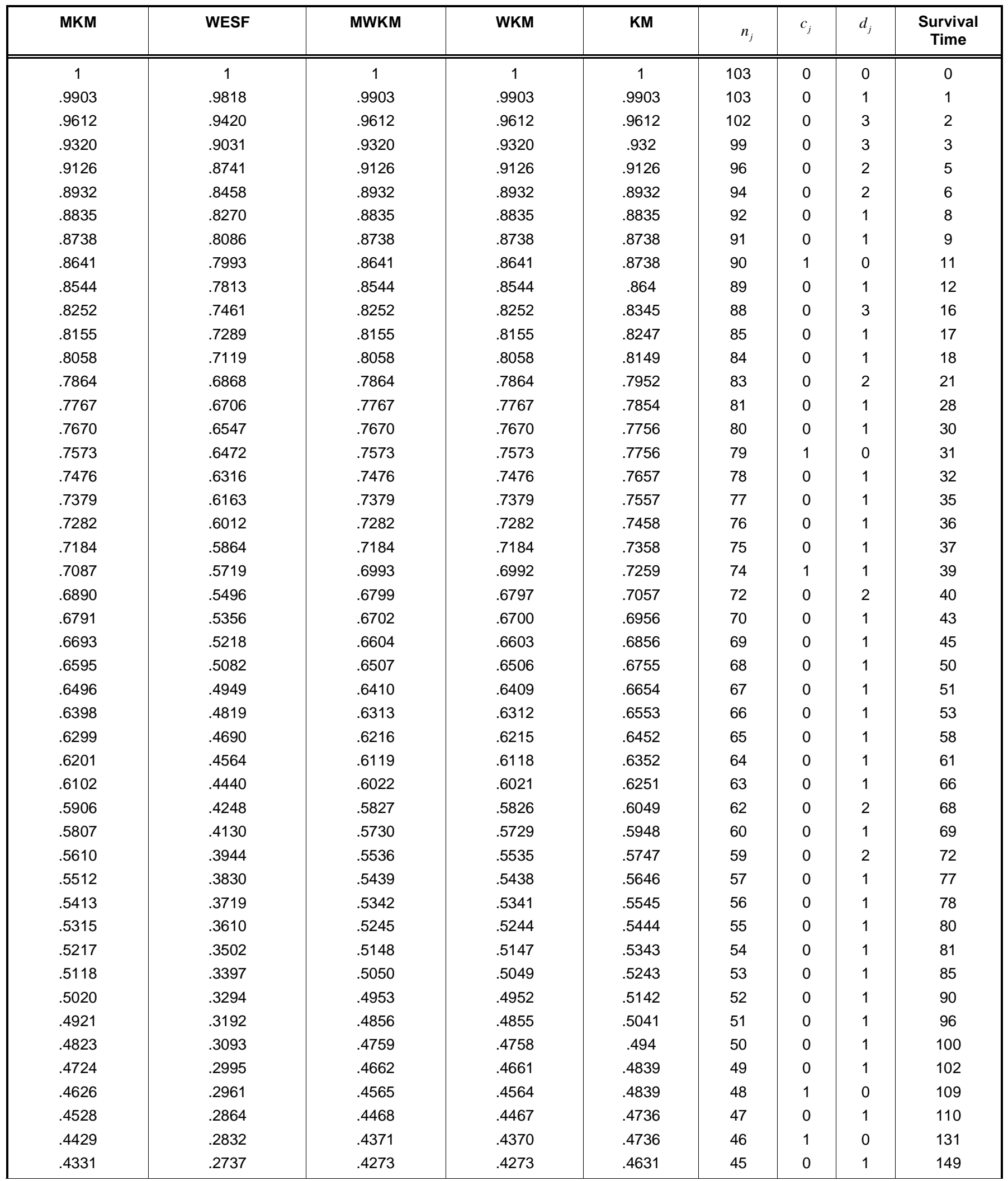




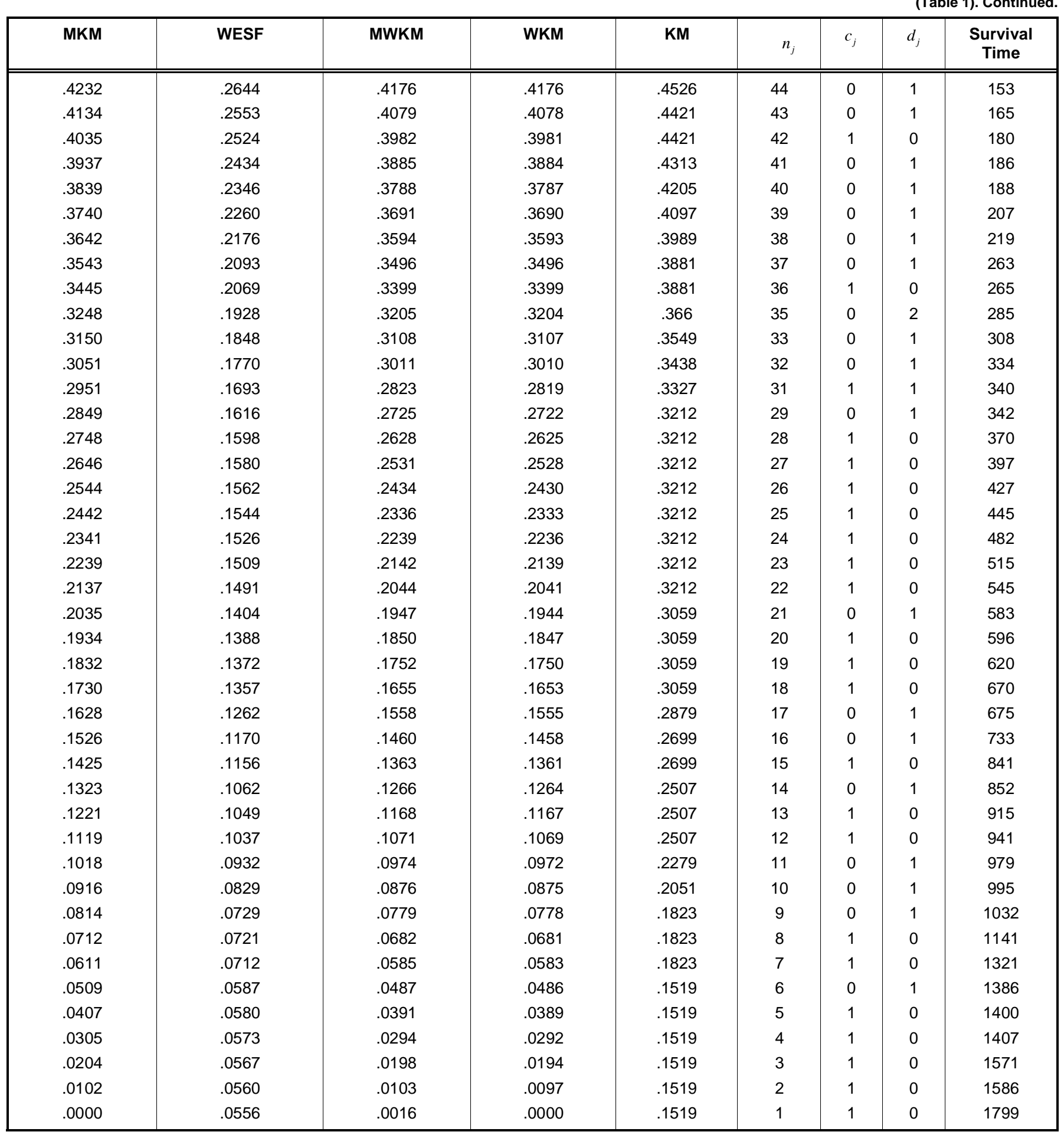
by:

And then the net probability of surviving is denoted

$p_{j}^{(1)}=1-q_{j}^{(1)}=\operatorname{Pr}$ \{a subject stays alive at time $t_{j}$ if the risk $R_{r}$ of being censored is eliminated\}

Note that $q_{j}^{(1)}$ and $q_{j}^{\prime(1)}$ are the crude and net probabilities of ding, respectively. In this paper, we consider two sources of decrement or competing risks, the risk of death $\left(R_{1}\right)$ and the risk of censoring $\left(R_{r}\right)$.

Taking this issue into consideration, subjects in a survival analysis are exposed to two risks; the risk of death and the risk of being censored. Thus, those subjects who die during the study would not be exposed to the risk of being censored anymore. It is incumbent on us to consider these subjects as people 
being exposed to just one risk and we have to calculate the net probability of dying for them. On the contrary, those subjects who are censored in the study are always exposed to death risk. Thus, the crude probability of censoring should be calculated for them. So the estimates of probability of ding in heavy censoring data are given as:

$$
q_{j}^{*(1)}=\left\{\begin{array}{lll}
q_{j}^{(1)} & \text { if } & d_{j} \neq 0 \\
q_{j}^{(r)} & \text { if } & d_{j}=0
\end{array} \quad \text {, where } q_{j}^{(1)}=\frac{q_{j}^{(1)}}{\left[1-0.5\left(q_{j}^{(r)}\right)\right]}\right.
$$

Where $q_{j}^{(1)}=d_{j} / n_{j}$ and $q_{j}^{(r)}=c_{j} / n_{j}$ are the crude probability of dying and the crude probability of censoring, respectively. And the proposed KaplanMeier estimator is then defined as:

$$
s^{*}(t)=\prod_{j: t_{(j)} \leq t}\left\{1-q_{j}^{*(1)}\right\}
$$

This proposed Kaplan-Meier estimator $\left(s^{*}(t)\right)$ is the same as the common Kaplan-Meier estimator when there is no censorship. Like Kaplan-Meier estimator, modified Kaplan-Meier estimator is a step function, with changes in survival probabilities at each failure/censored time.

\section{APPLICATION TO THE STANFORD HEART TRANSPLANT DATA}

In this study, the Stanford heart transplant data [18], which is a classic survival data set with time-dependent covariates, is reanalyzed. This well known data set has been considered and analyzed several times. We applied the Kaplan-Meier and Proposed method to data including censoring for finding the estimates of the survival curves, to ensure that the proposed method works better. The data set contains 103 patients from among them, 69 received transplants. The censoring rate is $27 \%$. The response variable is Survival time. The observed survival time is indicated to be censored or uncensored by the survival status.

The computation of survival probabilities for Stanford Heart Transplant Data using Kaplan-Meier (KM), Weighted Kaplan-Meier (WKM), Modified Weighted Kaplan-Meier (MWKM), Huang (WESF) methods and the proposed Kaplan-Meier estimation method based on competing (MKM) risks, has been presented in Table 1 . The survival computed, employing the above methods has been plotted in Figure 1.
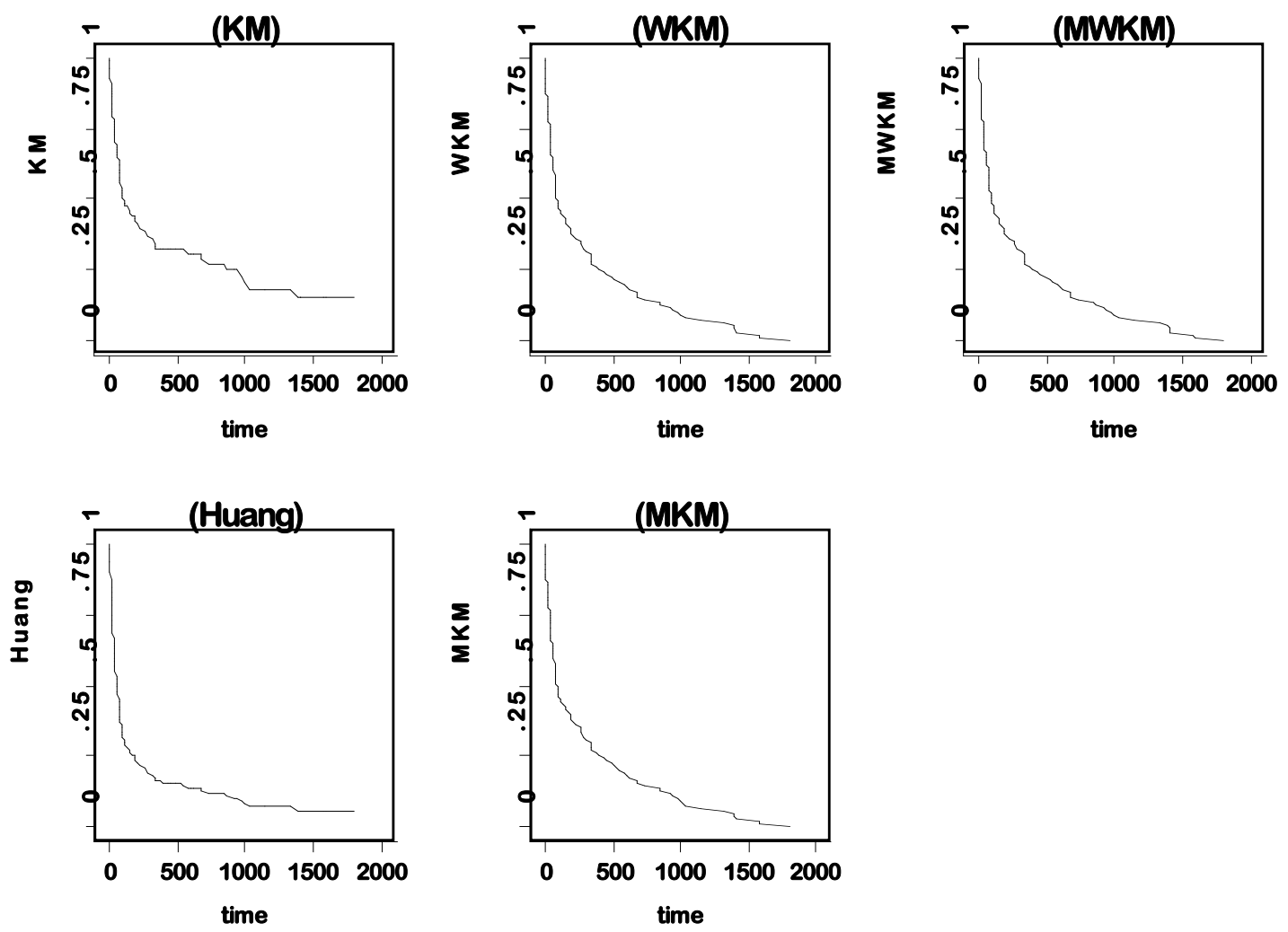

Figure 1: The Survival Curves for the HEART TRANSPLANT DATA by Kaplan-Meier (KM), Weighted Kaplan-Meier (WKM), Modified Weighted Kaplan-Meier (MWKM), Huang's Estimate and Modified Kaplan-Meier Estimator Based on Competing Risks (MKM). 
It is observed from Table 1 and the associated Figure 1 that the Kaplan-Meier method gives slightly a higher probability of Survival than the other methods at all of the censoring time points as expected. Moreover, in the tail part of the survival times the survival probability is higher than the others. Furthermore, as observed in Table $\mathbf{1}$ and Figure $\mathbf{1}$ our method, Weighted Kaplan-Meier and Modified Weighted Kaplan-Meier give better estimates in the case of heavy censoring. It is further noticed that the Modified Weighted Kaplan-Meier estimator is also in agreement with the Weighted Kaplan-Meier method. But the important point is that the Weighted Kaplan-Meier estimator gives zero weight to the last observation which is censored while the Modified Weighted KaplanMeier estimator gives it some nonzero weight and has a small probability of survival.

\section{CONCLUSION}

Kaplan and Meier proposed a nonparametric method for estimating the survival function, which has been recognized as a standard estimator for such probabilities [11]. This non-parametric estimator is still based on a number of assumptions. If one or more of these assumptions are violated, particularly in problems caused by a large number of censored values, the results of the analysis may become misleading. Heavy censoring affects the reliability of the Kaplan-Meier estimates [7, 11, 12]. In view of the heavy censoring (27\%) the Kaplan-Meier estimator is biased and overestimates the survival probabilities.

In this study a new method was presented based on competing risks theory. This proposed method was totally independent of any weights for censored observations, and it was just based on net and crude probabilities. This method is built on the assumption that the subjects in a survival analysis not only are exposed to death risk, but the risk of censoring. The subjects who die during the study are not exposed to censoring risk anymore. On the contrary, the censored subjects are always exposed to the risk of death. The Kaplan-Meier estimator always assumes that the death rate for subjects experiencing the event of interest is the same as the death rate in the population interest. This assumption is not met in cases in which censoring is too much in the study and this leads to bias in survival estimates. In Modified Kaplan-Meier, the problem of overestimating the survival estimators has been solved in cases in which censoring is too much. Moreover, in cases in which there are reasons other than censoring (death due to other reasons), this method can calculate the survival estimators.

This approach, like weighted Kaplan-Meier (WKM) and modified weighted Kaplan-Meier (MWKM,) gives unbiased estimate of the survival function at every point. Further, all these methods effectively deal with the tails of the survival distribution. As a result, the survival function curve touches the horizontal line, when the cohort ends even with a censored observation. Both the modified weighted Kaplan-Meier (MWKM) and weighted Kaplan-Meier (WKM) give same weight to all censored observations. They also give same probability of survival but the important point is that the WKM estimator gives zero weight to the last observation which is censored while the MWKM estimator gives it the same nonzero weight and has a small probability of survival.

Figure 1 shows that the majority of the death occurs at the early stages of survival time and higher survival times are mostly censored. It is observed that the Huang (WESF)' estimates underestimate the survival distribution at early stages and overestimate it at the tail points. Furthermore, as observed in Table 1, the three MKM, WKME and MWKME methods give far better results than other methods in case of heavy censoring. But either of the weights depends heavily on the event times and censoring distributions. Due to this fact, the weighted methods can have misleading results when the censoring patterns are different in the individual samples. Therefore it is concluded that the Modified Kaplan-Meier (MKE) MKM estimator considers not only the problem of heavy censoring but also the problem of weighted methods and competing risks in complicated data.

\section{REFERENCES}

[1] Andersen PK. Statistical models based on counting processes. Springer 1993 http://dx.doi.org/10.1007/978-1-4612-4348-9

[2] Young KD, Menegazzi JJ, Lewis RJ. Statistical methodology. Acad Emerg Med 1999; 6(3): 244-49. http://dx.doi.org/10.1111/i.1553-2712.1999.tb00165.x

[3] Klein JP, Moeschberger ML. Survival analysis: techniques for censored and truncated data. Springer 2003.

[4] Klein JP, Zhang MJ. Survival analysis, software. Wiley Online Library 2005.

[5] Murray S. Using Weighted Kaplan-Meier Statistics in Nonparametric Comparisons of Paired Censored Survival Outcomes. Biometrics 2001; 57(2): 361-68. http://dx.doi.org/10.1111/.0006-341X.2001.00361.x

[6] Hosmer Jr, DW, Lemeshow S, May S. Applied survival analysis: regression modeling of time to event data. WileyInterscience 2011; Vol. 618 
[7] Jan B, et al. Weighted Kaplan Meier estimation of survival function in heavy censoring. Pak J Statistics-All Series 2005; 21(1): 55.

[8] Jan B. Improved Inferences in the context of Survival/Failure Time, Ph. D Thesis University of Peshawar 2004.

[9] Shafiq M, Shah S, Alamgir M. Modified Weighted KaplanMeier Estimator. Pak J Statist Operat Res 2007; 3(1).

[10] Huang M. A weighted estimation method for survival function. Appl Math Sci 2008; 2(16): 753-62.

[11] Kaplan EL, Meier P. Nonparametric estimation from incomplete observations. J Am Statist Assoc 1958; 53(282): 457-81. http://dx.doi.org/10.1080/01621459.1958.10501452

[12] Kaplan E, Meier P. Nonparametric Estimation from Incomplete Observations, in Breakthroughs in Statistics. Springer 1992; pp. 319-337.

http://dx.doi.org/10.1007/978-1-4612-4380-9 25
[13] Chiang CL. A stochastic study of the life table and its applications. III. The follow-up study with the consideration of competing risks. Biometrics 1961; 17(1): 57-78. http://dx.doi.org/10.2307/2527496

[14] Chiang CL. Competing risks and conditional probabilities. Biometrics 1970; 767-776. http://dx.doi.org/10.2307/2528722

[15] Chiang CL. Competing risks in mortality analysis. Annual Rev Public Health 1991; 12(1): 281-307. http://dx.doi.org/10.1146/annurev.pu.12.050191.001433

[16] Bowers NL, et al., Actuarial mathematics. Society of Actuaries Itasca, III 1986; Vol. 2.

[17] Jordan C. Life Contingencies. Society of Actuaries 1975.

[18] Kalbfleisch JD, Prentice RL. The statistical analysis of failure time data. John Wiley \& Sons 2011; Vol. 360. 